\title{
The Cancer Cell Plasma Membrane Potentials As Energetic Equivalents to Astrophysical Properties
}

\author{
Michael A. Persinger*, Robert M. Lafrenie \\ Laurentian University, Sudbury, Ontario P3E 2C6, Canada \\ *E-mail address: mpersinger@laurentian.ca
}

\begin{abstract}
The primary physical and chemical parameters that define the hypopolarized plasma cell membrane of malignant (cancer) cells compared to non-malignant cells reflect universal characteristics. The median value for the resting membrane potential is the constant for the Nernst equation without reference to discrepancies in ion concentrations and is identical to Boltzmann energies at $37{ }^{\circ} \mathrm{C}$. The threshold energy defining space-time converges with access to entropic processes that are reflected in the morphology of cancer cells and tumors. Slowing of growth in cancer cell lines but not normal cells following exposure to weak $(\sim 1$ to $10 \mu \mathrm{T})$ patterned magnetic fields occurs when the energy induced within the cell corresponds to the energy equivalent of the hypopolarized membrane potential. The optimal temporal parameters for the efficacy of these fields can be derived from Hubble's parameter and the transform function for "noise" or "random" patterns within the system. Quantitative solutions and experimental data indicate that the cancer cell may be dominated by entropic process that can be attenuated or blocked by temporally-structured applied magnetic fields whose intensity matches the increment of energy associated with this threshold.
\end{abstract}

Keywords: kT boundary; magnetic energy; cancer cell; entropy; noise band; Hubble's parameter

\section{INTRODUCTION}

The plasma membrane potential of the mammalian cell has been considered the boundary condition that is primarily responsible for the disparity of charge producing the resting membrane potential. The classic Nernst equation [1] for this potential difference is:

$$
\mathrm{V}=\left[\mathrm{RT}\left(\mathrm{FZ}_{\mathrm{k}}\right)^{-1}\right] \cdot \log _{\mathrm{e}}\left[\mathrm{I}_{\mathrm{o}}\right] \cdot\left[\mathrm{I}_{\mathrm{i}}\right]^{-1}
$$

where $\mathrm{R}$ is the gas constant $\left(8.31 \mathrm{~J} \cdot \mathrm{T}^{-1} \mathrm{M}^{-1}\right), \mathrm{T}$ is temperature in degrees Kelvin, $\mathrm{F}$ is the Faraday constant $\left(9.65 \cdot 10^{5} \mathrm{~A} \cdot \mathrm{s}\right.$ per $\left.\mathrm{M}\right), \mathrm{M}$ is moles, $\mathrm{Z}$ is the valence of the ion, $\mathrm{I}_{\mathrm{o}}$ is the concentration of the ion along the outside of the membrane and $\mathrm{I}_{\mathrm{i}}$ is the concentration of that ion along the inside of the membrane. At $37{ }^{\circ} \mathrm{C}(310 \mathrm{~K})$ the solution for the constant, the first part of the term, is $26.7 \mathrm{mV}$. The source of the larger values for the resting membrane potentials, $-70 \mathrm{mV}$ to $-90 \mathrm{mV}$, for normal cells has been attributed to the disparity of concentration of ions, particularly potassium and chloride, between the outside and inside of the membrane. 
The recent review by Yang and Brackenbury [2] reiterated the frequently reported measurements that tumor cells display plasma membrane potentials that are more hypopolarized than normal or non-malignant cells. According to those authors the mean value for nine different classes of tumor cells is $\sim 24 \mathrm{mV}$ (range is between $-5 \mathrm{mV}$ and $-52 \mathrm{mV}$ ) which is within measurement error of $26.7 \mathrm{mV}$. In comparison the average resting membrane potential for eight different classes of non-tumor cells was $\sim-72 \mathrm{mV}$. The range was $-50 \mathrm{mV}$ (aorta smooth muscle) to $-93 \mathrm{mV}$ (skeletal muscle). Michael Levin's [3] review of eleven quiescent cell types from tissues such as thyroid, fat and kidney, ranged between -51 and -95 $\mathrm{mV}$. Adult stem cells' and tumor cells' resting membrane potentials ranged between -31 and and $-5 \mathrm{mV}$ with a clear cluster around $-25 \mathrm{mV}$.

The strong correlation between hypopolarization or "depolarization" and cancer cell proliferation has been known for more than 40 years. Cone [3] found that shifting the membrane potential of $\mathrm{CHO}$ cells from $-45 \mathrm{mV}$ to $-75 \mathrm{mV}$ produced mitotic cessation and blocked cell division. Hypopolarizing the cell membrane to $-10 \mathrm{mV}$ resumed cell cycle activity. We [4] have found that relatively weak, physiologically patterned magnetic fields slow the growth rates in several lines of malignant cells but does not affect non-malignant cell growth. Here we show that a physical-chemical approach from the perspective of increments of energy may explain this effect and suggest strategies for another form of treatment of malignant cells based upon principles and parameters shared by chemistry, physics and astronomy.

\section{CALCULATIONS}

If the cancer cell reflects a set or cluster of conditions that is grossly manifested as a hypopolarized membrane that approaches the Nernst solution $\left(\mathrm{RTF}^{-1}\right)$ for the common temperature without consideration of the ratios of internal to external membrane concentrations of ions, then the discernment of the energy associated with this band of potentials may be revealing for understanding mechanisms and possible treatment. At 26.7 $\mathrm{mV}$, the direct solution for potential difference at $37{ }^{\circ} \mathrm{C}$ from (1), the equivalent energy obtained by multiplying the unit charge of $1.6 \cdot 10^{-19} \mathrm{~A} \cdot \mathrm{s}$ is $4.27 \cdot 10^{-21} \mathrm{~J}$. This is the same value as the kT boundary or limit, where $\mathrm{k}$ is the Boltzmann constant $\left(1.38 \cdot 10^{-23} \mathrm{~J} \cdot \mathrm{T}^{-1}\right)$, for $37^{\circ} \mathrm{C}$. This boundary is frequently considered to be the "thermal noise" limit.

The potential difference of the cancer cell plasma membrane does not necessarily require the contribution from disparity of transmembrane charge. In other words the energy associated with the physical potential irrelevant of relative ion densities is equal to the energy which is the threshold from components derived from thermal agitation or Brownian movement. If membrane-related energies remained within this narrow band, information, measured in bits, would have the capacity to be dissipated or acquired according to the Landauer Limit of $\ln 2 \mathrm{kT}$, which is $2.95 \cdot 10^{-21} \mathrm{~J}$.

The dissipation of this energy contributes to or is highly correlated with the entropy or the disordered structure of the system. 


\section{MAGNETIC INTENSITY THRESHOLD}

Assuming the discrete energy associated with $26.7 \mathrm{mV}$ at $37{ }^{\circ} \mathrm{C}$ is completely coupled to the $4.27 \cdot 10^{-21} \mathrm{~J}$, then the equivalent magnetic field strength required to simulate this energy can be estimated by:

$$
\mathrm{B}=\sqrt{ }(\mathrm{J} \cdot 2 \mu) \cdot \mathrm{m}^{3}
$$

where $J$ is the energy, $\mu=$ magnetic permeability $\left(4 \pi \cdot 10^{-7} \mathrm{~N} \cdot \mathrm{A}^{-2}\right)$ and $\mathrm{m}^{3}$ refers to volume. The mouse melanoma B16 cell in cell culture on average displays a radius of $7 \mu \mathrm{m}$ such that the volume would be $1.44 \cdot 10^{-15} \mathrm{~m}^{3}$.

Hence the strength of magnetic field required to be juxtaposed upon the default resting membrane potential would be $\sim 2.7 \cdot 10^{-6} \mathrm{~T}$ or $27 \mathrm{mG}$. This is within the range of mean (RMS) intensities we have found delayed the growth of these cells specifically as well as other cancer cells by $\sim 50 \%$. The growth of normal or non-malignant cells was not affected.

\section{FREQUENCY BAND EFFICACY}

Physicochemical models for plasma cell membranes suggest they behave as a sheet of dipoles immersed within intrinsic strain from mutual interaction and from very local electric fields generated by cations within the structured space created by polyanionic glycoproteins of the outer membrane. The sialic acid side chains contain numerous fixed charged sites that exhibit a particularly high affinity for $\mathrm{H}^{+}$and $\mathrm{Ca}^{2+}$. Froehlich's [6] model allows energy storage and long-range coherence of energies along the major axis (perimeter) of the cell membrane because of recurrent hydrogen bonds and dipole interactions.

Grodsky's [7] model indicates that the liquid crystal features of the membrane resemble an antiferromagnetic crystal in an external magnetic field. Within a narrow range of fixed temperatures below a critical value the system displays phase transitions in response to sudden fluctuations in the surrounding electric field. Near a phase transition most of the total energy is contained within a narrow frequency band. A phase transition in a liquid crystal, which can be considered to be a feature of the fluid plasma membrane, is that point when configurational changes alter permeability.

According to Adey [8] the Boltzmann equation can be expressed in terms that model tissue as a low pass filter by:

$$
\mathrm{V}^{2}=4 \mathrm{kTB} \Omega
$$

where B is the frequency of the band width and $\Omega$ is resistance. Adey's interpretation was that equation (3) is a "transfer function" for the "noise" or random energy voltage that was a function of frequency band width and the specific resistance for the pathway. If the typical resistivity for interstitial fluid $(2 \Omega \cdot \mathrm{m})$ is applied and a band width only $2 \mathrm{~Hz}$ is assumed for $37^{\circ} \mathrm{C}$, the potential difference $(\mathrm{V})$ is $2.61 \cdot 10^{-10} \mathrm{~V} \cdot \mathrm{m}$.

Across the usual $10^{-8} \mathrm{~m}$ membrane, the equivalent potential difference is $\sim 26 \mathrm{mV}$. This solution indicates that when the plasma cell membrane behaves as a low pass filter, most of the energy is mediated within $\Delta \mathrm{f}=\sim 2 \mathrm{~Hz}$. If the functional distance is $100 \mathrm{~nm}$, the "extended" membrane that includes the "structure making" polyanionic nets that sequester $\mathrm{Ca}^{2+}$ and $\mathrm{H}^{+}$, the required $\Delta \mathrm{f}$ or "band" would increase to about $20 \mathrm{~Hz}$. 
This frequency band defines the shift in frequency modulation for the physiologicallypatterned magnetic fields that have been most effective in slowing the growth in vitro of four different types of cancer cell lines following one hour of exposure per day for 5 days. On the other hand the growth rates of six different lines of non-malignant cell exposed to these conditions were not affected. Repeated presentations of only the first component $(6 \mathrm{~Hz})$ or the second component $(25 \mathrm{~Hz})$ of the pattern did not produce the growth-inhibiting effect. The frequency modulation or shift in frequency within the band from $25 \mathrm{~Hz}$ to $6 \mathrm{~Hz}$ with each cycle was required.

Litovitz et al [9] experimentally demonstrated a threshold for temporal sensing of changing magnetic field structures by cells as measured by enhanced ornithine decarboxylase activity. They interrupted the field for brief intervals between 25 and $200 \mathrm{~ms}$ for one second throughout the exposure. Interruptions of more than $100 \mathrm{~ms}$ eliminated the field-induced enhancement of enzyme activity. In other words if the interruptions were less than $100 \mathrm{~ms}$ the cells did not "detect" the disruption as inferred by the maintained elevation in activity. These results indicate that millisecond-level continuities of magnetic field applications are important for the physical effects.

\section{EXPERIMENTAL EVIDENCE}

The most likely source of the discrepancy in resting plasma membrane potential between the non-malignant and malignant cell would be the persistent "opening" of potassium channels or the insertion of other types of channels (e.g., $\mathrm{Ca}^{2+}, \mathrm{Na}^{+}, \mathrm{H}^{+}$) that would increase the permeability. Assuming the difference between mean values of resting membrane potentials for malignant cells is $-26 \mathrm{mV}$ and that of the average non-malignant cell (neuron) is $-70 \mathrm{mV}$ there should be the replacement equivalent of about $44 \mathrm{mV}$ in cumulative ion channels.

If the singular resistance of a single open channel, $\sim 2 \cdot 10^{9} \Omega(2 \Omega$ divided by the width of a channel, $\sim 1 \mathrm{~nm}$ ) and the band width is $20 \mathrm{~Hz}$, the voltage for the transform is $\sim 2.6 \cdot 10^{-5}$ $\mathrm{V}$. To produce the cumulative change of $44 \mathrm{mV}$ would require the replacement of $\sim 1.7 \cdot 10^{3}$ channels. Yang et al [10 ], found that voltage-gated $\mathrm{Na}^{+}$channels are expressed not only in excitable cells like neurons but in several metastatic cells from cancers. Fraser et al [11] reiterated the lower (hypopolarized) resting membrane potentials for malignant breast cancer cells compared to normal breast cell lines. The primary voltage gated channel protein, $\mathrm{Na}_{\mathrm{v}} 1.5$ was expressed at levels $\sim 1000$ fold (i.e., $\sim 10^{3}$ ) higher in breast cancer cells than weakly metastatic cells.

In a similar manner changes in the expression of a wide variety of calcium channels have been linked to malignant behaviour. The increases in calcium currents have been associated with changes in cell migration and invasion. In particular, the voltage-dependent calcium channel, Cav3, is expressed on several cancers (prostrate, breast, melanoma) but not on non-malignant cells. Treatment of malignant cells with Cav3 channel blockers inhibits cell proliferation and migration.

This magnitude of up-regulation of $\mathrm{Na}^{+}$channels is convergent with the calculated predicted discrepancy in numbers of channels required to produce the disparity of resting membrane potentials between the two classes of cells. Increased $\mathrm{Na}^{+}$currents in breast cancer cell lines, enhanced migration, and infiltration could be attenuated by the sodium channel blocker phenytoin [10]. In fact blocking $\mathrm{Na}^{+}$currents carried by $\mathrm{Na}_{\mathrm{v}} 1.5$ in MDA-MB-231 
cells with tetrodotoxin, antibodies, or siRNA inhibited galvanotaxis, migration, and infiltration. However, cell-cell adhesion was enhanced.

It is relevant that voltage-gated sodium and calcium channels contribute to extension of cell processes and lateral motility. Greater fluidity of the plasma membrane can lead to deformability in melanoma cells. Ochalek et al [12] reported that these deformed cells displayed identical dimensions to other cells but were conspicuously metastatic. We cannot exclude the possibility, considering the elevated hydration states associated with $\mathrm{Na}^{+}$, that the phenomenon may reflect the characteristics of water [13 ].

\section{MALIGNANT CELLS AS ENTROPY-ACCELERATED CONDITIONS}

Matter and its complex organizations can be considered the form by which the final homogeneity of energy throughout space and time is delayed. From some perspectives the energy of the universe would have dissipated as soon as it began if it had not been for its representation in matter. The concept of entropy reflects a process rather than an event where a change from organized spatial patterns of matter to less organized matter occurs. The mechanism by which entropy is mediated usually includes the overinclusive term: random or "noise" which is the temporal equivalent of disorder in material (molecular and atomic) structures.

The second law of thermodynamics is often stated as "the universe tends towards chaos" [14], assuming the universe is an isolated system. If the assumptions of equilibrium are applied, entropy can be diminished, that is order can increase within highly local regions of the universe. The non-malignant cell can be considered a form of ordered, low-entropy structure [15]. However if Newton's third law predominates within an intricately connected system described by Mach's principle (that the momentum of any part of the universe is affected by all of its parts), for every formation of low-entropy structures (non-malignant cells) there must occur in non-locality a high entropy equivalent (malignant cells).

As reviewed and cogently described by Bizarri et al [16], the shape of the cell undergoes progressive changes that include altered cellular adhesive properties and the integrity of the cytoskeleton during transformation to neoplastic states. The morphology of the cell membrane displays an irregular, flattened and spindled profile that is reflected in the emergence of atypical microvilli and cell lability. At the larger level, the tumor, the irregular, non-smooth boundary is often a defining characteristic.

The shape of both the malignant cell and the tumor is more optimally described by fractal geometry. According to Bizarri et al [16] "high fractal dimension values are associated to a 'diffusive' shape, the form the cell acquires when it displays an invasive pattern, the stage that precedes metastatic spreading". Chemical treatments that reduce the fractal dimensions of at least one known cell line diminish malignancy.

We suggest that emergence of the plasma membrane potential around the kT boundary value allows access to the intrinsic "information" that is contained within the processes that mediate entropy. At present this process is assumed to be "random" or "noise" variations defined by in large part local energies within a narrow band of thermal conditions or the applied fields that simulate them. From this perspective the alterations in the shape of the cancer cell, that is increased fractal dimensions, do not reflect complexity. Instead it reflects the course towards entropy. Ultimately, if the circular shape of the membrane approached the mathematical limit of the smallest increment that could compose the circumference, complete disorder and hence homogeneity would emerge. 
The change begins with either the intrinsic (genetic) or adaptive (environmental) change of the plasma cell membrane that results in the hypopolarized membrane potential. Levin [4] has suggested that the physiological event, such depolarization into a particular voltagemembrane range, is responsible for the structural outcome. This bioelectrical signaling or "field" can be mediated through by any evolutionarily replaceable change in phenotype or channel formation that simply achieves a similar regulation of the specific membrane potential. In other words the electromagnetic field of the membrane controls the genetic expression that generates any number of classes of channels that will produce the optimal kTlimit potential energy.

The correlative alteration in the fluid phase-transitions of the membrane allows the deviation from simple symmetry. Because the experimental manipulation of the membrane potential within the range of $26 \mathrm{mV}$ facilitates cell division, the aberrant changes within the nucleus could be considered adaptive responses to this change. However the ultimate origin could be from the same source as that controlling "random" variation and entropy within the universe.

\section{ASTRONOMICAL PHYSICAL VARIABLES}

The Boltzmann formula for entropy results in an energetic value that was described initially as:

$$
\mathrm{S}=\mathrm{k} \cdot \ln \mathrm{W}
$$

where $\mathrm{W}$ is the number of microstates within a macrostate. Within the total boundary condition, the universe, one solution is that the numbers of microstates are photon units. Hence, there are $\sim 10^{104}$ photons within that macrostate. This value is derived from the mass of the universe of $\sim 10^{52} \mathrm{~kg}$ [17] divided by the upper limit for the estimated rest mass of $10^{-52} \mathrm{~kg}$ for a photon [18]. The $\ln$ of $10^{104}$ is 239.46; consequently the entropy threshold for this system would be $3.30 \cdot 10^{-21} \mathrm{~J} \cdot \mathrm{T}^{-1}$. This value is within the (mid) range between the energy for a bit of information to dissipated into entropy upon erasure or convergence of operations according to the Landauer solution and both the kT boundary and energetic equivalents for the default Nernst equation.

Assuming the current average background temperature of the universe to be consistent with the Cosmic Microwave Background $(2.73 \mathrm{~K})$ the threshold to access entropy within the entire system would be $\sim 0.9 \cdot 10^{-20} \mathrm{~J}$ or $56 \mathrm{mV}$ when considering the effect upon a single unit charge. This "access" value is within error of measurement of the value of the resting membrane potentials that separate non-malignant and malignant cells.

The frequency equivalence of $\mathrm{CMB}$ is $160.2 \mathrm{GHz}$ or $1.6 \cdot 10^{11} \mathrm{~Hz}$. This frequency is within the order of magnitude described by Froehlich [6] to describe the energy storage and long range coherence due to recurrent hydrogen bonds and dipole interactions within the plasma cell membrane. They would occur, according to Froehlich, within the range of 100 to $1000 \mathrm{GHz}$.

The second universal factor involves absolute expansion of space according to Hubble's constant. Persinger and Koren [19] first reported that for an intermediate range for this parameter, $75 \mathrm{~km} \cdot \mathrm{s}^{-1}$ per MParsec $\left(3.1 \cdot 10^{22} \mathrm{~m}\right)$, the value is $2.4 \cdot 10^{-18} \mathrm{~s}^{-1}$. The length of a proton was assumed to be twice the Compton radius of $1.3 \cdot 10^{-15} \mathrm{~m}$. Consequently, the proton's velocity of expansion would be $6.2 \cdot 10^{-33} \mathrm{~m} \cdot \mathrm{s}^{-1}$. 
The time required to expand one Planck's Length would be $1.6 \cdot 10^{-35} \mathrm{~m}$ divided by $6.2 \cdot 10^{-33} \mathrm{~m} \cdot \mathrm{s}^{-1}$ or about $2.6 \mathrm{~ms}$. More recent calculations based on current Hubble estimates [20] indicate a value closer to $3 \mathrm{~ms}$. This is the point duration of the magnetic fields that generated the most effective diminishment of cancer cell growth.

Subsequent experiments indicated that reactions that are mediated by $\mathrm{H}^{+}$were most affected by $3 \mathrm{~ms}$ point durations. These included shifts in $\mathrm{pH}$ in spring water by changing the proportions and dynamics of the hydronium ion [13]. Shifts occurred in non-local volumes of spring water when local volumes of spring water were injected with small amounts of a weak acid assuming both volumes shared the same circularly rotating magnetic field. The changes in the non-local volume were in the order of $0.2 \mathrm{pH}$ units. That protons can act as neurotransmitters in increments of $0.2 \mathrm{pH}$ units has been reported by Highstein et al [21]. They considered the relative latency for the accumulation of $\mathrm{H}^{+}$to produce the effect to be optimal for tonic signals such as from gravity.

The final potential universal contributor may emerge from the zero-point energy of a quantized field. This defines the Casimir effect, which in its simplest form is "the interaction of a pair of neutral, parallel conducting planes due to the disturbance of the vacuum of the electromagnetic field" [22]. Only the surface layer of atoms, analogous to the single layer of charge that contributes to the resting membrane potential [23], is considered a continuum that interacts with the electromagnetic field.

Boundaries are concentrations of external fields that polarize the vacuum of a quantized field. When the boundary condition depends upon time, energy is transferred from the external field to vacuum oscillations, often considered "virtual particles". There is a transformation, hypothetically at least, to real particles. In other words when the boundary condition is a function of time particle creation occurs. The Casimir pressure is optimally described by:

$$
\mathrm{P}=\left(\pi^{2} 240^{-1}\right) \cdot(\hbar \mathrm{c}) \cdot \mathrm{a}^{-4}
$$

where $\mathrm{P}=$ pressure, $\hbar=$ the modified Planck's constant $\left(1.05 \cdot 10^{-34} \mathrm{~J} \cdot \mathrm{s}\right)$, c is the velocity of light in a vacuum $\left(3 \cdot 10^{8} \mathrm{~m} \cdot \mathrm{s}^{-1}\right)$ and " $\mathrm{a}$ " is the separation between the "plates".

If we assume the pressure is the universal value that defines variants of the cosmological constant, $1.5 \cdot 10^{-10} \mathrm{~Pa}$ (from the product of $1.67 \cdot 10^{-27} \mathrm{~kg} \cdot \mathrm{m}^{-3}$ and the square of the velocity of light $\left.\left(9 \cdot 10^{16} \mathrm{~m}^{2} \cdot \mathrm{s}^{-2}\right)\right)$, then $\mathrm{a}^{4}$ can be obtained. The value is $8.71 \cdot 10^{-18} \mathrm{~m}^{4}$ and the linear equivalent is $54 \mu \mathrm{m}$. The equivalent frequency when divided into the velocity of light is $0.55 \cdot 10^{13} \mathrm{~Hz}$ and the equivalent energy after multiplication by Planck's standard constant $\left(6.626 \cdot 10^{-34} \mathrm{~J} \cdot \mathrm{s}\right)$ is $3.65 \cdot 10^{-21} \mathrm{~J}$. The voltage equivalent is $\sim 23 \mathrm{mV}$. For the solution to be $26 \mathrm{mV}$, only the square of the coefficient for the average proton density $\left(2.78 \cdot 10^{-27}\right.$ $\mathrm{kg} \cdot \mathrm{m}^{-3}$ ) is required. This conspicuous convergence suggests that the energy equivalent of the resting membrane potential for malignant cells might be coupled to the average universal pressure.

\section{IMPLICATIONS FOR TREATMENT}

The matching of the energy associated with the kT boundary for $37{ }^{\circ} \mathrm{C}$, the $\mathrm{RT} \cdot \mathrm{F}^{-1}$ solution for the Nernst coefficient and the energy density produced by exposure of a cell to a physiologically patterned magnetic field that delays growth in several cancer cell lines but not normal cells indicates the presence of a narrow band with which external influence is 
possible. The proposed process is that the application of the temporally-patterned magnetic field within the specific band of intensity (and hence energy) alters the "random" or "noise" mechanisms that promote entropy. This process may define the classic cancer cell and may be the consequence of the second law of thermodynamics which states functionally that for ever local region of order there must be induced, somewhere in space, a comparable area of entropy.

However the primary determinant of the energy within the cell is its volume. This means that cells, even within the same cell line, that exhibit different average volumes would respond optimally to the specific intensity that presents the optimal energy values. For B16 melanoma mouse cells whose widths can very from 10 to $25 \mu \mathrm{m}$, this means that the optimal average (RMS) magnetic field would vary by discrete values in order to be most effective.. Because cells within a plate also show a range, not all cells would be equally affected. This may explain the consistent observation that growth rate in about 1 million cells is slowed by about $40 \%$ compared to non-exposed cells.

The optimal point duration, the time each amplitude of magnetic field that contributes to the pattern generated by computer software to the application geometry (pairs of solenoids or coils), has been $3 \mathrm{~ms}$. Presentations of the same pattern magnetic field with point durations of $1 \mathrm{~ms}, 2 \mathrm{~ms}, 4 \mathrm{~ms}$ or $5 \mathrm{~ms}$ is not effective [5]. This effect can be visualized with fluorescent calcium infiltration in cell cultures that begins about $15 \mathrm{~min}$ after field onset and is optimal after about $1 \mathrm{hr}$ of exposure. The effect is blocked by calcium T-channel compounds, suggesting this channel is mediating the magnetic field pattern's inhibiting effect.

If the magnetic field is inhibiting the effect of the hypopolarization by simulating the effects of deficient numbers of selective potassium (or chloride) channels that contribute to the passive resting membrane potential, then the opening of calcium T-channels might be expected. Potassium dependent calcium channels are known to mediate critical changes within the cell. Simulation of potassium channels by the specific intensity and pattern of field might produce a virtual coupling by which T-channels are opened.

As aptly described by Cifra et al [24], Froehlich's model of coherent longitudinal (around the perimeter of the cell) vibrations of electrically polarized structures, such as those found in most proteins, is dependent upon metabolic energy. These two features, coherent longitudinal vibrations and derivation from metabolic energy, have two important implications from a physical-chemistry and even astronomical perspective.

Coherent vibrations within the $10^{11}$ to $10^{12} \mathrm{~Hz}$ range around a cell would be within the range of $\mathrm{CMB}$ for the entire universe. If one assumes that the nature of "entropy" and the random processes by which it is obtained involve this band then any cell that acquires this coherence might become resonant. The net result could be the shift in channel morphologies or functions that lead to diminished plasma membrane potential and the plurality of intracellular changes that follow. The application of a repeated complex but temporally structured magnetic field within the optimal intensity range would interfere with this resonance by producing a "temporal structure".

Vibrations moving around a closed geometry, such as circle, imply angular momentum and intrinsic acceleration. Shared angular momentum is a proposed mechanism for excessive correlation or entanglement between systems over space. Dotta et al [25] found that the cell may display a "membrane magnetic moment", analogous to the magnetic moment of the Bohr magneton, with which applied magnetic fields can interact. The optimal intensity of the applied magnetic field produced a discrete amount of energy (photons) that is within the visible wavelength that could be detected by photomultiplier units. 
Dotta and Persinger [26] found that two photoluminescent $\left(\mathrm{H}_{2} \mathrm{O}_{2}+\mathrm{HClO}\right.$, hypochlorite) reactions separated by non traditional distances but each placed in the center of a rotating magnetic field with coupled changing angular velocities showed conspicuous excessive correlation. If the two separate dishes were injected with a quantity of reactant and at the same time both shared the same temporal configuration of circular magnetic fields, the photon emissions doubled. In other words, the photon energies responded as if the two separate loci were in the same space, that is, the same space received twice the quantity of reactant.

Considering the evidence that: 1) the movement of charges due to lateral diffusion within a plasma cell membrane is effectively a rotating magnetic field, and, 2 photon emissions can exhibit "excess correlation" between two non-local areas that share similar field configurations, the potential for information exchange between cells over large expanses of the body become probable.

This may allow an alternative process, traditionally attributed to migrational-based metastasis, by which "action at a distance" might occur as well as a method for minimizing the probability.

This approach would be consistent with Levin's review of the concept that cancer reflects cells that elude the "morphogenetic" field of the organism and that cancer is not a genetic disease of specific loci but an anomaly of cells states. They can be described by the same intrinsic processes, such as attractors [25] that can define astronomical phenomena.

\section{CONCLUSIONS}

The bimodal distribution of the resting membrane potentials of different classes of "cancer" cells and non-malignant cells shows that the median plasma membrane potentials of the former distribute around $26.7 \mathrm{mV}$, the constant for the Nernst equation. The energy associated with this value is identical to the threshold where thermal "oscillations" and "random" processes dominate the system.

Applied weak magnetic fields whose energies within the cell volume juxtapose the intrinsic energies delay cell division and require a frequency band that corresponds to a resistance across the $100 \mathrm{~nm}$ space associated with the plasma membrane and attached polyanionic nets that sequester $\mathrm{Ca}^{2+}$ and other cations.

The convergent solutions from values that define universal parameters reiterate the general physical bases for biological activity and suggest that the cancer cell phenomena reflect an entropic direction that has been enhanced and supported by cell structure. Applications of the appropriate temporally patterned magnetic fields slow the processes that lead to entropy and hence aberrant cell growth is delayed.

\section{ACKNOWLEDGEMENTS}

We thank Dr. W. E. Bosarge, Jr. CEO, Capital Technologies, Inc. for his support of these concepts and applications. 


\section{References}

[1] J. W. Woodbury, in T. C. Ruch, H. D. Patton, J. W. Woodbury, A. L. Towe (eds), Neurophysiology, W. B. Saunders, Philadelphia, 1968, 1-25.

[2] M. Yang, W. J. Brackenbury, Frontiers in Physiology 4 (2013) 1-10.

[3] M. Levin, BioEssays 34(3) (2012) 205-217.

[4] C. D. Cone, Oncology 24 (1970) 438-470.

[5] C. Buckner, Effects of Electromagnetic Fields on Biological Processes are Spatial and Temporal Dependent, Ph.D. Thesis Biomolecular Sciences, Laurentian University, Sudbury, 2011.

[6] H. Froelich, International Journal of Quantum Chemistry 2 (1968) 641-649.

[7] L. T. Grodsky, Mathematical and Biological Sciences 28 (1976) 191-219.

[8] W. R. Adey, S. M. Bawin, Neurosciences Research Program Bulletin 15 (1977) 1-129.

[9] T. A. Litowitz, M. Penafiel, D. Krause, D. Zhang, J. M. Mullins, Bioelectromagnetics 18 (1997) 388-395.

[10] M. Yang, D. J. Kozminski, L. A. Wold, R. Modak, J. D. Calhoun, L. L.Isom, W. J. Brackenbury, Breast Cancer Research Treatments 134 (2012) 603-615.

[11] S. P. Fraser, J. K. Diss, A-M. Chioni, et al, Clinical Cancer Research 11 (2005) 5381-5389.

[12] T. Ochalek, F. J. Nordt, K. Tullberg, M. M. Burger, Cancer Research 48 (1988) 5124-5128.

[13] N. J. Murugan, L. M. Karbowski, M. A. Persinger, Water 6 (2014) 45-60.

[14] G. Woodbury, Physical Chemistry, Brooks/Cole Publishing Co, Pacific Grove, 1997.

[15] B. Albert, A. Johnson, J. Lewis, M. Raff, K. Roberts, P. Walter, Molecular biology of the cell, Gs Garland Science, N.Y, 2002.

[16] M. Bizzarii, A. Pasqualato, A. Cucina, V. Pasta, Histology and Histopathology 28 (2013) 155-174.

[17] M. A. Persinger, Journal of Physics, Astrophysics and Physical Cosmology 3 (2009) $1-3$.

[18] L-C Tu, J. Luo, G. T Gillies, Reports on Progress in Physics 68 (2005) 77-130.

[19] M. A. Persinger, S. A. Koren, International Journal of Neuroscience 117 (2007) 157-175.

[20] S. A. Koren, B. T. Dotta, M. A. Persinger, The Open Astronomy Journal 7 (2104) 1-6. 
[21] S. M. Highstein, G. R. Holstein, M. A. Mann, R. D. Rabbit, PNAS Early Edition, 2014, www.pnas.org/cgi/doi/10.1073/pnas.1319561111.

[22] M. Bordag, U. Mohideen, V. M. Mostepanenko, Physics Reports 353 (2001) 1-205.

[23] M. A. Persinger, Neuroscience and Biobehavioral Rev 36 (2012) 2334-2338.

[24] M. Cifra, J. Z. Fields, A. Farhadi, Progress in Biophysics and Molecular Biology 105 (2011) 223-246.

[25] B. T. Dotta, R. M. Lafrenie, L. M. Karbowski, M. A. Persinger, General Physiology and Biophyics 33 (2014) 63-73.

[26] B. T. Dotta, M. A. Persinger, Journal of Biophysical Chemistry 3 (2012) 72-80.

[27] M. Levin, BioSystems 109 (2012) 243-261. 